\title{
Enfermedad de Weber-Christian
}

\author{
Marisol Avendaño B. ${ }^{1}$; Arnoldo Quezada L. ${ }^{1}$; Samuel Benveniste D. ${ }^{1,2}$; \\ Carmen Salgado M., ${ }^{1,2}$ Jorge Vergara $C^{2}$
}

\section{Weber-Christian disease}

\begin{abstract}
A tourteen month old infant was admitted for evaluation because of continuous high fever and an indurated nodular Iesion at the left thigh of one month course. After admittance palnful inflamatory subcutaneous nodules appeared in the face and trunk, these were acompanied by enlarged cervical lymph nodes and hepatomegaly. Histological evaluation of the sk in biopsy showed destruction of subcutaneous tissue, foamy celis, vasculitis and polymorphonuclear leukocyte infiltration: histiocytic proliferation in the lymph nodes and steatosis in the liver biopsv. Osteoarticular infection, cellulitis, sepsis, tuberculosis, collagen disease, and malignancies of hematologic origin were all ruled out. Response to treatmen with prednisone was excellent and the patient has been asymptomatic along a one year follow up period.

iKev words: pannıculitis, fever of unknown origin. Weber-Christian disease.)
\end{abstract}

Las pariculitis son enfermedades caracterizadas por la presencia de un proceso inflamatorio del tejido adiposo subcutáneo, secundario a una alteración vascular local o producida por daño directo del adipocito. La paniculitis de Weber-Christian o paniculitis nodular recidivante no supurativa fue descrita por primera vez en 1925 por Weber en adultos y en 1928 Christian le agregó el adjetivo "febril"1, 2.

Clínicamente, el sindrome de Weber-Christian se caracteriza por la presencia de nódulos y placas en el tejido subcutáneo, que excepcionalmente son exudativas. En su evolución natural los nódulos aparecen bruscamente, persisten semanas y se resuelven para reaparecer en brotes recurrentes a lo largo de 2 a 5 años. Los enfermos presentan fiebre, generalmente alta, mialgias, trastornos digestivos, con o sin compromiso de órganos (principalmente páncreas, hígado y mesenterio); si existe compromiso visceral, es de peor pronóstico y se denomina enfermedad de Weber-Christian $^{3,4}$. El sindrome afecta con mayor frecuencia a mujeres obesas entre $20 \mathrm{y}$ 60 años de edad, pero se ha descrito aun en el período neonatal. Se han publicado aproximadamente 60 casos en menores de 12 años ${ }^{1}$.

1. Departamento de Pediatría y Cirugía Infantil Sur, Facultad de Medicina, Universidad de Chile.

2. Servicio de Pediatría, Hospital Exequiel González Cortés.
El objeto de esta comunicación es difundir las caracteristicas de esta enfermedad prácticamente desconocida en el ámbito pediátrico, discutir el diagnóstico diferencial y destacar la importancia del estudjo histológico en su identificación.

\section{Caso Clínico}

Varón, nacido por cesárea, peso de naciniento: $3.340 \mathrm{~g}$, período de recién nacido nomal, lactancia materna durante 10 meses. Sin antecedentes mórbidos de importancia. Ingresó al servicio de enfermedades infecciosas del Hospital Exequiel González Cortés a los 14 meses de edad por la aparición de una placa indurada con signos intlamatorios en la cara externa del muslo izquierdo acompañada de fiebre alta, de hasta $41^{\circ} \mathrm{C}$, ambos de un mes de evolución, por lo que había recibjdo cloxacilina oral en foma irregular durante 21 dias sin resultados favorables. No tenía antecedentes de traumatismo, infecciones ni uso de otros medicamentos. Al ingresar estaba quejumbroso, temperatura rectal: $40,3^{\circ} \mathrm{C}$, estado de nutrición normal; en el tercio medio de cara externa de muslo izquierdo presentaba una placa eritematosa violácea de $8 \mathrm{~cm}$ de diámetro, con calor local y una solución central de continujdad, de la que drenaba un exudado de aspecto caseoso. Hematocrito $30 \%$, hemoglobina $10,2 \mathrm{~g} / \mathrm{dl}$, leucocitos $4.700 \mathrm{x} \mathrm{mm}^{3}$, con $36 \%$ de baciliformes y velocidad de sedimentación eritrocitaria $33 \mathrm{~mm} / \mathrm{h}$, patrón que se mantuvo durante toda la hospitalización. Después de descartar una infección osteoarticular, se interpretó su problema como celulitis y se trató con ampicilina y cloxacjlina endovenosa, evolucionando con una curva térmica de tipo séptico $y$ hemocultivos siempre negativos. El esquema antibiótico inicial se cambió en varjas opor- 
tunidades dc acuerdo a la sensibilidad de diferentes gérmenes aislados de cultivos locales de la lesión de la piel (Pseudomona aeruginosa, Staphylococcus aureus meticilina resistente, Escherichia coli, Klebsiella pneumoniae). A fines de la segunda semana de hospitalización se realizó exploración quirúrgica de la lesión del musio sin encontrar pus y se tomó bjopsia de piel cuyo estudio histológico mostró signos de inflamación crónica reagudizada, con necrosis del tejido graso. Por la misma época se detectó hepatomegalia de $3 \mathrm{~cm}$ bajo el reborde costal, confirmsda mediante ecografía abdominal, sin alteraciones de los diferentes indicadores de función hepática. En el mieloguama no se encontraron signos de enfermedades hemato-oncológicas. El cultivo de médula ósea, los estudios de tuberculosis, factor reumatoideo, anticuerpos antinucleares y factor C3 del complemento sérico dieron resultados negativos. En la cuarta semana de hospitalización el paciente seguía febril, pero con buen estado general. En esa etapa aparecieron aproximadamente 10 nodulos subcutáneos en el tronco y la cara, de 1 a $2 \mathrm{~cm}$ de diámetro, sensibles, eritematosos (figura 1), sinultáneamente con adenomegalias cervicales, a raíz de lo cual se hicieron biopsias de uno de los nódulos, de un ganglio y del hígado, por punción. En el nóđulo subcutáneo se encontró extensa destrucción đel tejido celular subcutáneo, presencia de células espumosas, vasculitis e infiltración inflamatoria por leucocitos polımorfonucleares (figura 2a, b). En el ganglio había proliferación histiocitaria y en el hígado esteatosis del tipo "de gota grande". La amilasemia era normal y no fue posible determinar lipasa pancreática en el suero.

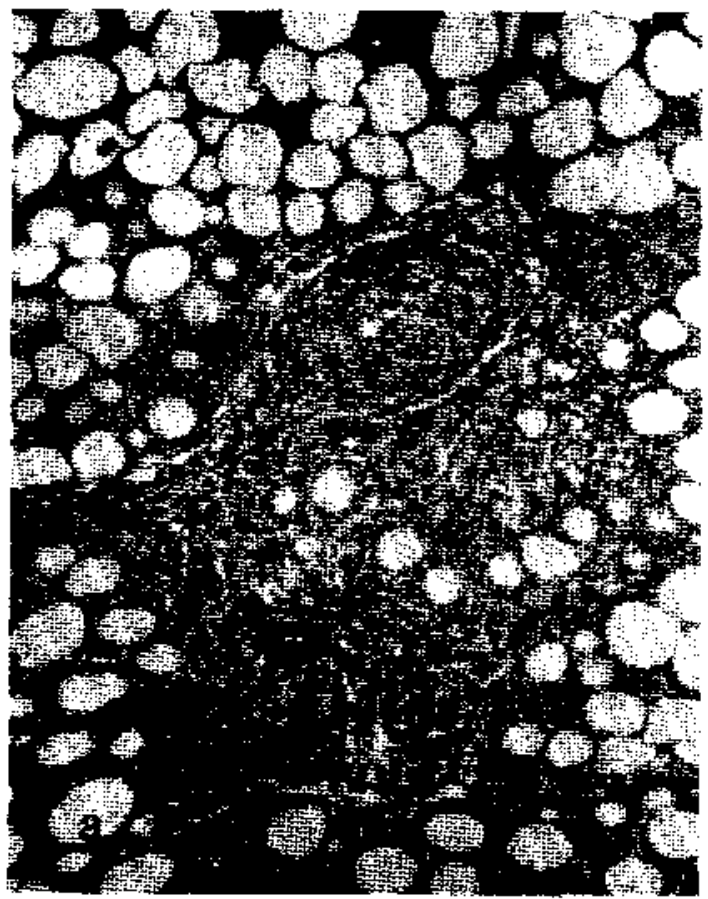

Fig. 2: Biopsia de la pjel del mismo niño a las 8 gemanas de evolución. a: Necrosis del tejido adiposo subcutáneo, vasculitis e infiltrado con leucocitos po-

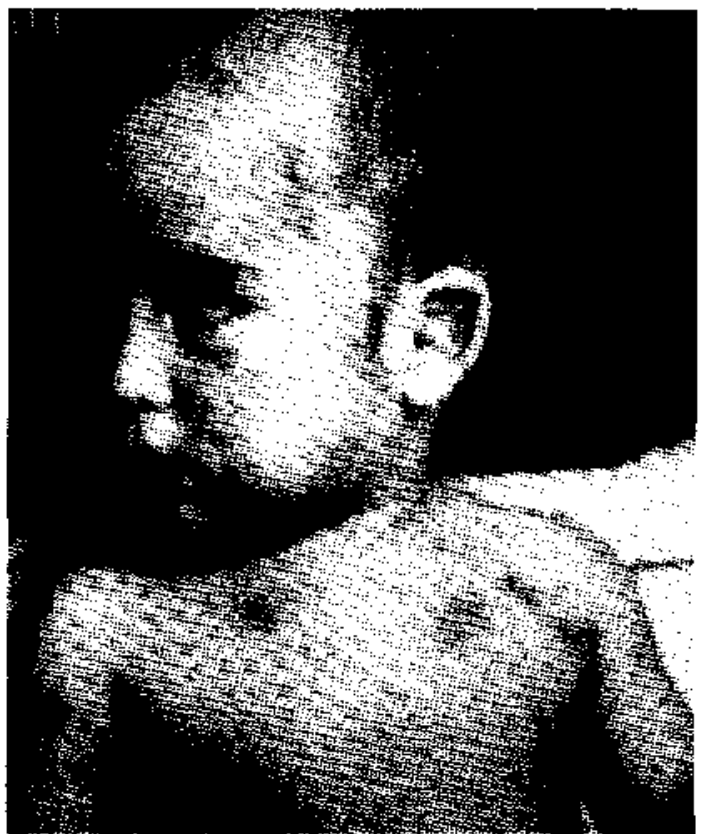

Fig. 1: Paciente de 14 meses de edad. Fiebre $y$ lesión eritematosa, violácea con necrosis central en muslo izquierdo, de 8 semanas de duración. La fotografía muestra nódulos subcutáneos sensibles, eritematosos, de cara y tronco aparecidos en la octava semana.

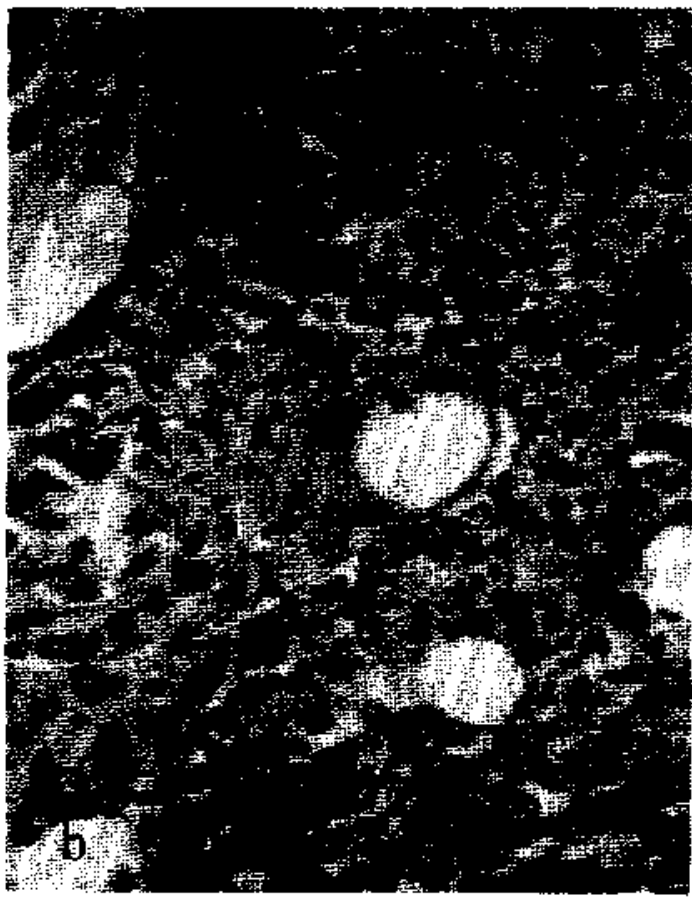

limorfonucleares (tinción hematoxilina-eosina, $350 \mathrm{x}$ ). b: Proliferación de histiocitos y fagocitos de adipositos necrotizados. 
Sumando estos resultados a la clínica, se diagnosticó enfermedad de Weber-Christian $y$ se inicio tratamiento con prednisona oral ( $1 \mathrm{mg} \cdot \mathrm{kg}$ en días alternos), en coincidencia con lo cual se produjo descenso de la temperatura, desaparición de los nódulos subcutáneos y disminución de la lesión del muslo sin que se resolviêse totalmente la solución de continuidad cutánea, que requirió reparación quirúrgica (figura $3 a, b$ ). A los 12 meses de tratamiento esteroidal en dosis decrecientes, ef niffo no ha presentado recurrencias, no hay evidencias de compromiso visceral ni efectos secundarios de los corticoides.

\section{Discusión}

Las paniculitis son poco frecuentes, más aún en los niffos. En este caso, en particular el diag. nóstico diferencial se debe hacer con todas las enfermedades que se manifiestan como sindrome febril prolongado (principalmente enfermedades infecciosas, oncológicas y del mesénquima) y con otras paniculitis agudas ${ }^{5}$, como el eritema nodoso, eritema indurado o paniculitis por frío. Sin embargo, la que más se asemeja a la enfer. medad de Weber-Christian es la paniculitis asociada a enfermedad pancreática crónica, que también evoluciona en brotes recurrentes y se debe a la liberación al torrente circulatorio de enzimas pancreáticas que destruyen el tejido graso periférico. En este enfermo la amilasa plasmática fue normal. La paniculitis de la enfer. medad pancreática crónica es propia de los

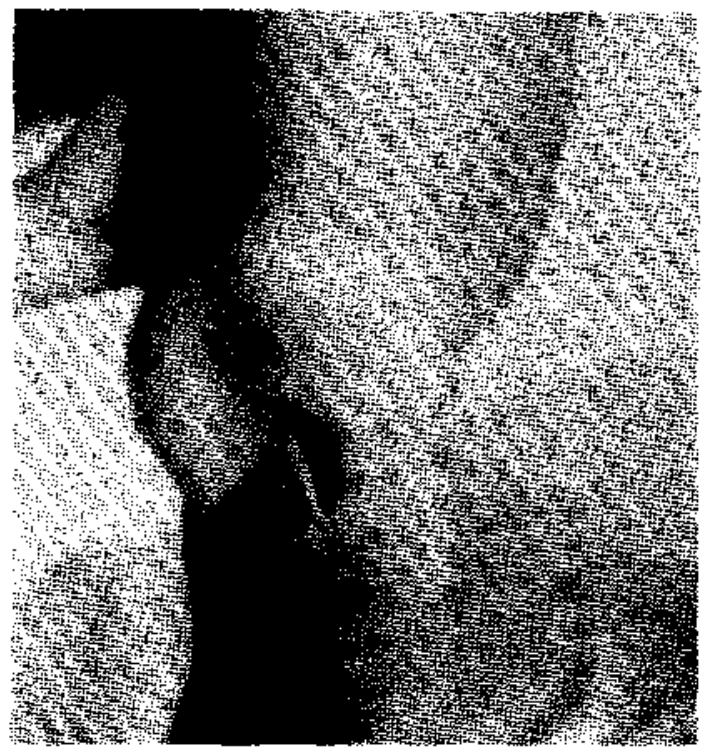

Fig. 3: Aspecto de la piel del paciente después de iniciar tratamiento con prednisona. a: Desaparición de adultos y se diferencia histológicanente de la enfermedad de Weber-Christian porque no existe vasculitis.

En la enfermedad de Weber-Christian la histología es fundamental para el diagnóstico. Su evolución presenta tres fases tipicamente diferenciadas y se caracteriza por la existencia de paniculitis y vasculitis. En la fase 1 existe degene. raciōn de los adipocitos con ruptura de sus membranas citoplasmáticas y pérdida de coloración de sus núcleos, por lo que se les ha denominado células fantasmas. Además hay infiltración celular por leucocitos polimorfonucleares, constitu. yendo una reacción inflamatoria inespecífica. En la fase 2 se observa proliferación de histiocitos, los que fagocitan a los adipocitos destruidos y dan origen a las llamadas células espumosas. Finalmente, la fase 3 se caracteriza por formación de tejido fibroso, con fibroblastos entremezclados con linfocitos y cẻlulas plasmáticas. Si existe compromiso del dermis y la epidermis (úlcera), hay fenómenos de colicuación generalmente asociados con aiteración vascular. En este enfermo la biopsia de piel fue concordante con la fase evolutiva 2 , ya que había histiocitos fagocitando células adiposas destruidas (figura 2b), además de los fenómenos de vasculitis e infiltración por polimorfonucleares, sin que se detectasen elementos de fibrosis. Las alteraciones ganglionares y hepáticas confirmaron el compromiso visceral.

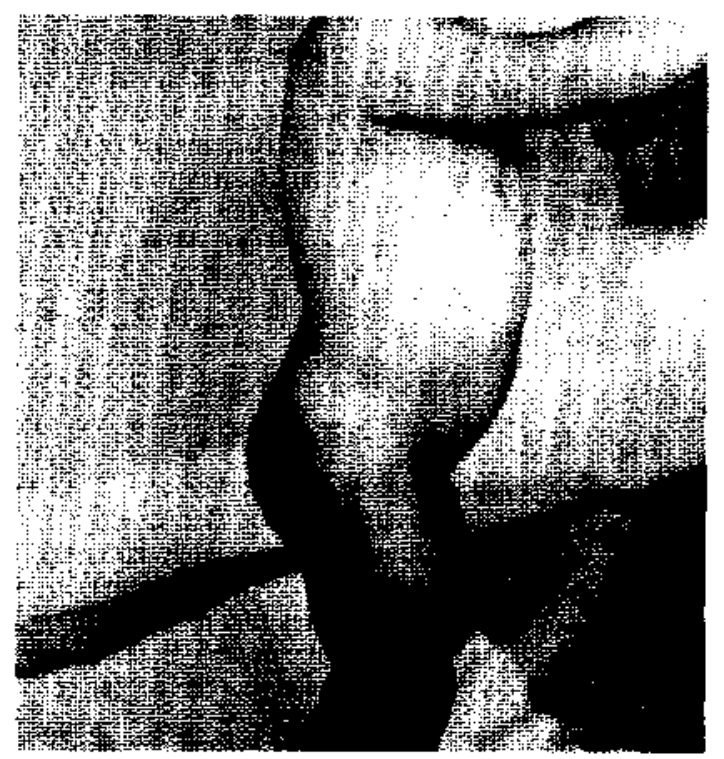

los nódulos, persistiendo lesión necrótica del muslo. b: Después de la reparación quirứrgica. 
La etiologia de esta enfermedad no ha sido dilucidada, pero se presume que tiene origen autoinmune por la asociación de este síndrome con enfermedades como el lupus eritematoso, la artritis reumatoidea $y$ el déficit de Alfa-1antitripsina ${ }^{6-9}$. Se ha sugerido que por algún mecanismo se alterarían los antígenos del tejido adiposo provocando la formación de autoanticuerpos, de los cuales se han demostrado leucoaglutirinas circulantes ${ }^{10}$

También se ha planteado que los linfocitos $T$ tendrian un papel importante en la patogenia de esta enfermedad, ya que se ha observado remisión de los brotes en relación a disminución de su población y reaparición de las manifestaciones clínicas al normalizarse aquélla ${ }^{1}$. En los casos que comienzan a temprana edad se ha postulado la existencia de una anormalidad congénita que determina reacciones inflamatorias exageradas, tanto locales y generales, dirigidas principalmente contra el tejido graso, pero que pueden comprometer otros órganos ${ }^{11}$.

Se han ensayado diferentes modalidades de tratamiento $0^{8,10-13}$, entre ellas antiinflamatorios no esteroidales, antibióticos y cloroquina, pero los inmunosupresores han dado los mejores resultados, lo cual avala aún más la probable etiología inmunitaria. Se han usado corticoides, azathioprina y ciclofosfamida. Estas dos últimas drogas se emplean cuando no hay respuesta a los corticoides o cuando hay evidencia de compromiso visceral importante y la evolución de la enfermedad sugiere un curso potencialmente letal. En el caso que se comenta, con signos de afección visceral, la respuesta a la prednisona fue excelente y la evolución posterior ha sido muy satisfactoria.

\section{Resumen}

Un niño de 14 meses de edad ingresó por presentar placa indurada en la piel del muslo izquierdo y fiebre alta de un mes de evolu. ción. Durante su hospitalización aparecieron nódulos subcutáneos sensibles con características inflamatorias en cara y tronco, adenopatías cervicales y hepatomegalia. En la biopsia de la piel habia extensa destrucción del tejido celular subcutáneo, células espumosas, vasculitis e infiltración por leucocitos polimorfonucleares; en la de ganglio linfático se comprobó proliferación histiocitaria y en una muestra de hígado obtenida por punción se encontró evidencia de esteatosis. Se descartaron osteoartritis, celulitis, septicemia, tuberculosis, enfermedad del colágeno y reoplasia linforreticular. Recibió tratamiento con prednisona con excelente respuesta. Se ha mantenjdo asintornatico a lo largo de 12 meses de seguimiento.

(Palabras clave: panjculitis, sindrome febril prolongado, enfermedad de Weber-Christian.)

\section{Referencias}

1. Hendricks, W.M.; Ahmad, M,; Grat, E.: WeberChristian syndrome in infancy. Br J Dermatol 1978; 98: 175-185.

2. Robbins, S.L.: Enfermedades Generales. Enfermedad de Weber-Christian. En: Robbins, S.L. Patología extruciural y funcional. México: Nueva Editorial Interamericana, 1975: 304-305.

3. Edge, $J$; Dunger, D.B.; Dillon, M.J.: Webet-Christjan Pannjculitis and chronic active hepatitis. Eur J Pediatr 1986; 145: 227.229.

4. Stack, J.; Heffernon, S.J.; Dervan, P.: Ennis, J.Y.: Inaging of a case of sclerosing panniculitis. Br J Radiol 1986; 59: 1119-1121.

5. Färstrōm, L.; Winkelmann, R.K.: Acute Panniculitis. A Clinical and Histopathologic study of 34 cases. Arch Dermatol 1977; 113: 909-917.

6. Allen-Mersch, T.G.: Weber-Christian panniculitis and autoimmune disease: A case report. J Clin Pathol 1976; 29 : 144-149.

7. Rubinstein, H.M.; Joffer, A.M; Kudmo, J.C. et ad.: Alpha 1-antitrypsin deficiency with severe panniculitis. Ann Intern Med 1977; 86: 742-744.

8. Strunk, R.W.; Schel, W.M.: Cyclophosphamide therapy for Weber-Christian disease associated with Alpha 1-antitrypsin deficiency. South Med J $1986 ; 79: 1425-1427$.

9. Breif, S.N.; Clork, P.; Robinson, J.P.: Luckhurst, E.; Dawkins, R.L.; Penny, R.: Familial Occurrence of alpha 1 -antitrypsin deficiency and WeberChristian disease. Arch Dermatol 1983; 119: 198-202.

10. Fye, K.H.; Tolal, N.; Enfermedades reumáticas. Paniculitis recurrente. En: Fudenberg, H.H. Inmunología clínjca, México: Editorial Manual Moderno, 1982; 480 .

11. Sorensen, R.U.; Abramowsky, C.R.; Stern, R.C.: Ten-Year course of early-onset Weber-Christian syndrome with recurrent Preumonia: A suggestion for pathogenesis. Pediatrics 1986; 78: 115 120 .

12. Hotta, T.; Wakamatsu, Y.; Matsumura, N. ef al.: Azathioprine-Induced remission in Weber-Christian disease. South Med J 1981; 74: 234-237.

13. Leider, M.: Paniculitis nodular recjdivante, febril y no supurativa. En: Leider, M. Dermatología Pediátrica, Buenos Aires: Editorial Bibliográfíca Argentina, $1969 ; 496$. 